\title{
Relationship between phase shift and energy dissipation in tapping-mode scanning force microscopy
}

\author{
Javier Tamayo and Ricardo García ${ }^{a}$ \\ Instituto de Microelectrónica de Madrid, CSIC, 28760 Tres Cantos, Madrid, Spain
}

(Received 20 July 1998; accepted for publication 16 September 1998)

\begin{abstract}
Force curves taken during a load-unload cycle show the presence of a hysteresis loop. The area enclosed by the loop is used to measure the energy dissipated by the tip-sample interaction in tapping-mode scanning force microscopy. The values of the energy loss obtained from force curves are compared with the results derived from a model based on phase shift measurements. The agreement obtained between both methods demonstrates that for the same operating conditions, the higher the phase shift the larger the amount of energy dissipated by the tip-sample interaction. It also confirms the prediction that phase-contrast images can only arise if there are tip-sample inelastic interactions. (C) 1998 American Institute of Physics. [S0003-6951(98)03646-8]
\end{abstract}

The dynamic operation of a scanning force microscope (SFM) was introduced to minimize tip-sample deformation and, in some cases, to improve spatial resolution with respect to contact SFM operation. ${ }^{1-3}$ The tapping mode, ${ }^{4}$ also known as intermittent contact mode, is arguably the most extended dynamic SFM mode. There, the cantilever-tip ensemble is oscillated at a frequency close to its resonance. At one end of each oscillation the tip strikes the sample. The sample is imaged while the oscillation amplitude, usually called tapping amplitude, is kept at a constant value. The very small lateral force exerted on the sample explains its ability to image compliant materials. ${ }^{5}$

Tapping-mode SFM is also relevant because it has introduced phase contrast imaging. There, the measurement of the difference between the phase angle of the excitation signal and the phase angle of the cantilever response is used to map compositional variations in heterogeneous samples. ${ }^{5-7}$ This mode is usually called phase-contrast or phase shift imaging. Despite its wide experimental use, only a few models have been provided to explain the origin and the meaning of the contrast obtained in phase images. ${ }^{8-11}$

In previous papers, we have shown that phase-contrast images are associated with the presence of tip-sample inelastic interactions. ${ }^{10,11}$ Recently, Cleveland et al. have proposed an expression for the power dissipated by the cantilever-tip ensemble. ${ }^{12}$ This expression establishes a relationship between the sine of the phase shift and the power loss. However, a direct comparison between the energy dissipated and the results deduced from phase shift measurements has not been provided.

In this letter, force curves taken during a loadingunloading cycle are used to measure the energy dissipated per period in tapping mode SFM. The dominant feature revealing nonconservative processes is the presence of a hysteresis loop in a representation of the force versus tip displacement. The area enclosed by the loop represents the energy dissipated per cycle. This energy is compared with the values obtained from a model based on phase shift mea-

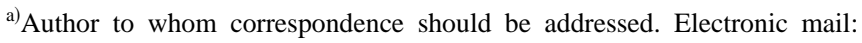
rgarcia@imm.cnm.csic.es
}

surements. The agreement obtained between both results confirms the prediction that phase-contrast images can only arise if there are tip-sample inelastic interactions. ${ }^{10}$ It also supports the interpretation of phase images as maps of energy dissipation. ${ }^{12}$

The experiments were performed using commercial SFM electronics and software. All measurements have been made in air at relative humidity of $35 \%$. A silicon cantilever with a spring constant $k$ of $45 \mathrm{~N} / \mathrm{m}$, resonance frequency $f_{0}$ of $356 \mathrm{kHz}$ and quality factor $Q$ of $270 \pm 40$ was used. The cantilever was excited at its resonance frequency.

We have chosen patches of purple membranes (PM) deposited on highly oriented pyrolitic graphite (HOPG) as the sample to perform the experimental measurements. This sample provides a heterogeneous surface formed by protein membranes (low elastic modulus) surrounded by the graphite surface (large elastic modulus).

A $10 \mu \mathrm{l}$ drop of an aqueous suspension containing 0.1 $\mathrm{mg} / \mathrm{ml}$ of $\mathrm{PM}$ was sprayed onto a piece $(5 \mathrm{~mm} \times 5 \mathrm{~mm})$ of HOPG. With no further preparation the sample was introduced into the SFM chamber. Experimental details about sample preparation can be found elsewhere. ${ }^{13}$

Figure 1 shows a phase image of a PM patch on HOPG. The diagonal lines show the presence of monoatomic and multiatomic steps on the graphite. A large membrane occupies the central region of the image while two small fragments are seen on the right. The phase shift on the HOPG is about $10^{\circ}$ higher than on the membrane. The phase shift is also independent of the thickness of the membrane.

To measure the energy dissipated by the tip-sample forces during tapping-mode, SFM, force curves were taken. First, the cantilever's deflection dependence on the tipsample separation was recorded (feedback mechanism was turned off). The force is deduced by multiplying the cantilever's deflection by its spring constant. The deflection was measured while the cantilever-tip ensemble was approached and then retracted from the sample at $40 \mathrm{~Hz}$ (loadingunloading cycle). We have assumed that the force curves do not depend on the loading-unloading frequency.

Figure 2 shows the force curves obtained in the regions marked by crosses in Fig. 1. The attractive forces are larger 


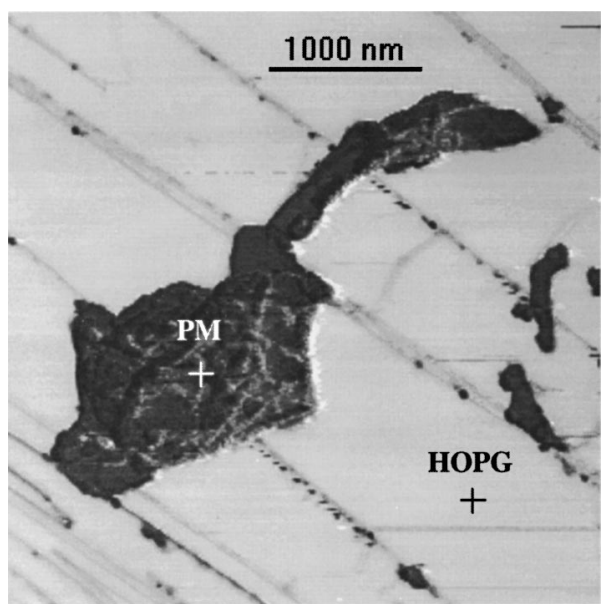

FIG. 1. Phase-contrast image of a purple membrane on graphite. The phase shift is $10.5 \pm 0.5^{\circ}$ higher on graphite than on the membrane. This shift is independent of the number of membranes piled up. The thickness of a single membrane is about $5 \mathrm{~nm} . A_{0}=43 \mathrm{~nm}, A_{t}=34 \mathrm{~nm}, k=45 \mathrm{~N} / \mathrm{m}, f=f_{0}$ $=356 \mathrm{KHz}$, and $Q=270$. The crosses mark the sites where the force curves were measured.

on the graphite regions than on the PM, however they show similar features. The load-unload curves overlap in the repulsive force region. This suggests a repulsive tip-sample interaction dominated by the elastic properties of the sample (the elastic modulus of the tip is several times higher than the modulus of the sample). Overall, these force curves are characteristic of tip-sample interactions dominated by long-range forces with neither mechanical instabilities associated with the cantilever nor long-range plastic deformations. ${ }^{14}$

Major differences between loading and unloading force curves are found in the region dominated by attractive forces. This is indicative that the energy loss is dominated by hysteresis in the adhesion energy and/or capillary forces. The
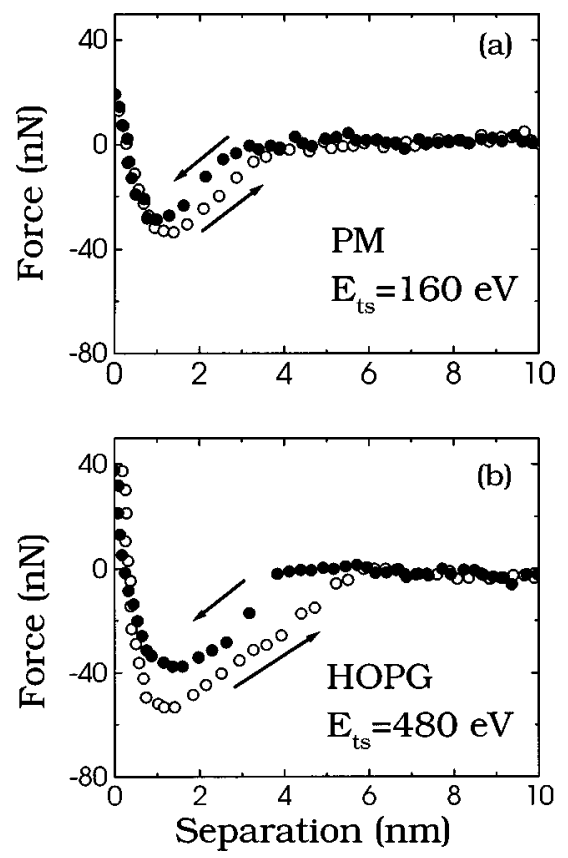

FIG. 2. Force vs tip-sample separation on a purple membrane (a) and graphite (b). The arrows indicate the direction of the relative tip-sample displacement. The area enclosed between load-unload curves is the energy dissipated. The energies dissipated on the purple membrane and on the graphite

are $160 \pm 20$ and $480 \pm 20 \mathrm{eV}$, respectively.
Downloaded $15 \mathrm{Feb} 2010$ to 161.111 .180 .191$. Redistribution subject to AlP license or copyright; see http://apl.aip.org/apl/copyright.jsp

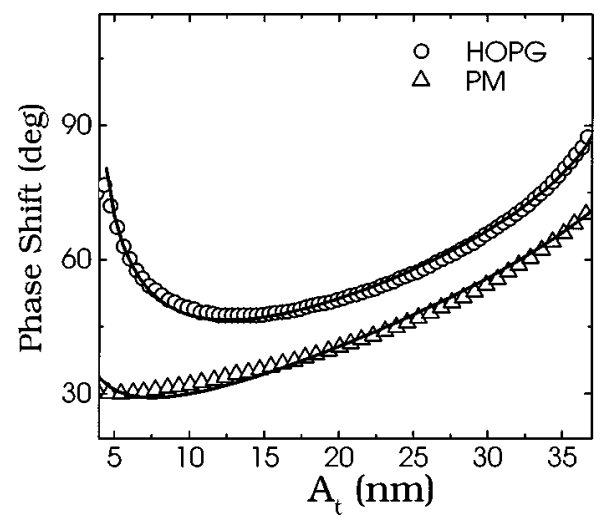

FIG. 3. Phase shift dependence on the tapping amplitude for the membrane (triangles) and for the graphite (circles). The solid lines are the results obtained from Eq. (2) Instrumental data, $f / f_{0}=1, A_{0}=43 \mathrm{~nm}, k=45 \mathrm{~N} / \mathrm{m}$, and $Q=270$.

area enclosed by the force curves represents the energy dissipated by the tip-sample interaction, $E_{\mathrm{ts}}$. This gives $E_{\mathrm{ts}}$ of $480 \pm 20 \mathrm{eV}$ for HOPG and $160 \pm 20 \mathrm{eV}$ for PM. Those values are about two orders of magnitude larger than the energy needed to break a single covalent bond. These experiments have been performed with tips of curvature radius of about $60 \mathrm{~nm}$. The loss of energy is spread out over a contact area involving about 2000 atoms. As a consequence, the energy dissipated per bond is a about $0.1 \mathrm{eV}$ for PM and $0.25 \mathrm{eV}$ for graphite. It seems reasonable to assume that energy is being dissipated without material removal.

Next, we have determined the energy deduced from phase shift measurements. A relationship between the phase shift and the energy dissipated in tapping mode SFM is obtained by considering that in the steady state the external energy ( $\left.E_{\text {ext }}\right)$ supplied to the cantilever must equal the energy dissipated via hydrodynamic viscous interactions with the environment (air) and by the tip-sample interaction

$$
E_{\text {ext }}=E_{\text {air }}+E_{\text {dis }} \text {. }
$$

From this equation and under the assumption of a sinusoidal cantilever response, ${ }^{12}$ an expression that relates the phase shift angle $(\varphi)$ to the energy dissipated by the tipsample interactions $E_{\text {dis }}$ per period is deduced

$$
\sin \varphi=\frac{f}{f_{0}} \frac{A_{t}(f)}{A_{0}}+\frac{Q E_{\mathrm{dis}}}{\pi k A_{0} A_{t}(f)},
$$

where $f$ and $f_{0}$ are the excitation and natural frequencies of the cantilever, respectively. $A_{t}$ and $A_{0}$ are the tapping and the free amplitude, respectively.

The phase shift versus the tapping amplitude is plotted in Fig. 3. The experiments were performed with a free amplitude of $A_{0}=43 \mathrm{~nm}$. The range of $A_{t}$ shown involves tipsample intermittent contact. The measurements were performed on the regions marked by crosses in Fig. 1. The experimental data were derived from the phase shift versus distance and amplitude versus distance curves taken simultaneously (not shown). The phase shift decreases with $A_{t}$ in both PM and HOPG [first term of Eq. (2)], however this decrease is larger on the membrane. This reflects the influence of the second term of Eq. (2).

The continuous line represents the theoretical simulation obtained from Eq. (2). The fitting parameter used was $E_{\text {dis }}$. 
Values of $E_{\mathrm{dis}}=180 \mathrm{eV}$ and $E_{\mathrm{dis}}=510 \mathrm{eV}$ were obtained for the membrane and the graphite, respectively. It is worth noting that the numerical as well as the shape agreement obtained between the experimental and simulated data over the tapping amplitude range explored. More importantly, the above estimation is close to the data obtained from force curves $\left(E_{\mathrm{ts}}=160 \mathrm{eV}\right.$ and $E_{\mathrm{ts}}=480 \mathrm{eV}$, respectively). This agreement is a direct demonstration of the relationship between the phase shift and the energy loss established in Eq. (2). Phase shift and force curves were also taken in 64 different regions of the image to check for the homogeneity of the data. Similar results were obtained.

The local processes responsible for the hysteresis observed in the force curves are still not identified. It is likely in the contribution from capillary forces and/or adhesion energy hysteresis, ${ }^{15}$ however, neither the experimental measurements nor the model made any assumptions about the nature of the inelastic processes. As a consequence, the conclusions derived here have a general character.

In summary, we have measured the energy dissipated during tapping-mode SFM by analyzing the loadingunloading force hysteresis. The values of the energy obtained from force curves are very similar to those deduced from a model based on phase shift measurements. First, this confirms that phase images can be interpreted as maps of energy dissipation. For the same tapping mode SFM conditions, the higher the phase shift, the larger the amount of energy dissi- pated. Second, in the absence of plastic deformation, the hysteresis in the force curves is the major source of phase contrast.

This work has been supported by the Dirección General de Investigación Científica y Técnica of Spain (PB94-0016) and the European Commission (BICEPS, BIO4-CT97-2112)

${ }^{1}$ Y. Martin, C. C. Williams, and H. K. Wickramasinghe, J. Appl. Phys. 61, 4723 (1987).

${ }^{2}$ D. Anselmetti, R. Lüthi, E. Meyer, T. Richmond, M. Dreier, J. E. Frommer, and H.-J. Güntherodt, Nanotechnology 5, 87 (1994).

${ }^{3}$ F. J. Giessibl, Science 267, 68 (1995).

${ }^{4}$ Q. Zhong, D. Imniss, K. Kjoller, and V. B. Elings, Surf. Sci. 290, L688 (1993).

${ }^{5}$ J. Tamayo and R. García, Langmuir 12, 4430 (1996).

${ }^{6}$ K. Sasaki, Y. Koike, H. Azehara, H. Horaki, and M. Fujihira, Appl. Phys. A: Mater. Sci. Process. 66, 1275 (1998).

${ }^{7}$ G. Bar, Y. Thomann, and M.-H. Whangbo, Langmuir 14, 1219 (1998).

${ }^{8}$ R. G. Winkler, J. P. Spatz, S. Sheiko, M. Möller, R. Reineker, and O. Marti, Phys. Rev. B 54, 8908 (1996).

${ }^{9}$ S. N. Magonov, V. Elings, and M.-H. Whangbo, Surf. Sci. 375, L385 (1997).

${ }^{10}$ J. Tamayo and R. García, Appl. Phys. Lett. 71, 2394 (1997).

${ }^{11}$ R. García, J. Tamayo, M. Calleja, and F. García, Appl. Phys. A: Mater. Sci. Process. 66, 309 (1998).

${ }^{12}$ J. P. Cleveland, B. Anczykowski, A. E. Schmid, and V. Elings, Appl. Phys. Lett. 72, 2613 (1998).

${ }^{13}$ R. García, J. Tamayo, J. M. Soler, and C. Bustamante, Langmuir 11, 2109 (1995).

${ }^{14}$ N. A. Burham, R. J. Colton, and H. Pollock, Nanotechnology 4, 64 (1993).

${ }^{15}$ M. K. Chaudhury, Mater. Sci. Eng., R. 16, 97 (1996). 